
stratégies de formation

\title{
Les rapports images/textes
}

Interviewé par Ch. QUENTIN

\section{Bernard Marquetti}

\section{(2) OpenEdition}

\section{Journals}

Édition électronique

URL : http://journals.openedition.org/edc/2866

DOI : 10.4000/edc.2866

ISSN : 2101-0366

Éditeur

Université Lille-3

Édition imprimée

Date de publication : 1 mai 1989

Pagination : 121-124

ISSN : 1270-6841

Référence électronique

Bernard Marquetti, «Les rapports images/textes », Études de communication [En ligne], 10 | 1989, mis en ligne le 12 février 2012, consulté le 05 mai 2019. URL : http://journals.openedition.org/edc/2866 ; DOI : 10.4000/edc. 2866

Ce document a été généré automatiquement le 5 mai 2019.

(c) Tous droits réservés 


\title{
Les rapports images/textes
}

\author{
Interviewé par Ch. QUENTIN
}

Bernard Marquetti

1 Les rapports du texte et de l'image à l'intérieur du reportage sont régis de façon très précise, chaque élément compte. Ainsi une musique «bien choisie " peut être déjà un commentaire.

2 «La télévision c'est de l'image » mais à l'image il faut ajouter le son ambiance, le son interview, les témoignages et le texte du journaliste.

3 Le tout constitue un seul et unique message, que le journaliste a pour tâche de mettre en ordre, de gouverner. On dit du texte par rapport à l'image qu'il a deux fonctions :

- une fonction d'identification

- et une fonction de relais.

4 Lorsque le texte cadre bien à l'image, il ancre cette image ; c'est l'exemple du Président Mitterrand, qui arrive à Moscou ; l'image de cette arrivée paraît sur l'écran, l'ancrage est produit par ce texte : « le Président de la république est reçu par Monsieur Gorbatchev » (si l'on voit Gorbatchev) mais si l'on dit : c'est le 4ème voyage du président Mitterrand, on nie l'image diffusée simultanément. Si je prends un cas de figure où l'image est signifiante ; elle représente la scène à laquelle on assiste, l'ancrage advient lorsque cette scène est identifiée par le journaliste qui dit «voilà de quoi nous parlons ». La fonction relais intervient par rapport à la polysémie de l'image et permet d'en préciser le sens. Différentes figures peuvent se combiner :

- La redondance : on répète l'image pour que le message passe mieux.

- Le contrepoinc le plus délicat à utiliser pour un journaliste. Il peut provoquer un excès de sens et conduire à caricaturer le message. C'est un moyen très fort mais rarement utilisé. Quelquefois, en sport, quand un footballer marque contre son camp, le commentaire reprend «but magnifique»; c'est une complicité du commentateur qui ne prête pas à conséquence.

- La complémentarité est le moyen le plus courant. C'est ce que le journaliste va dire et que ne dit pas l'image. 

reportage sur une prison; l'image montre en plongée la cour d'une prison avec un personnage au centre, un grillage quadrille le premier plan de cette image. pour éviter les évasions... ». Voilà donc comment chemine le texte par rapport à l'image : comme une sorte de courbe sinusoïde parallèle au défilement plan des images ; ce qui donne une identification de l'image par le commentaire dès les premières secondes, puis un éloignement apporté par une information complémentaire, puis à nouveau une identification, etc... Le meilleur commentaire étant celui qui retombe le plus souvent dans l'image et, à l'inverse, le moins bon, celui qui poursuit sa route seul, sans tenir compte de l'image.

Dans cette situation le téléspectateur peut faire des rapprochements de sens, mais c'est le travail du journaliste de faire en sorte que tout soit compris d'emblée par le téléspectateur, afin qu'il n'ait pas de perturbations à l'écoute. L'écoute pour une chaîne de télévision, c'est la règle d'or, il faut donc absolument retenir les gens par un discours unique texte/image bien imbriqué et sans dysfonctionnements.

Pour le journaliste une question se pose sans cesse : « qu'est-ce que je veux dire? » ou bien encore : « je suis là pour dire quoi ? ». Il doit se tenir à un seul angle d'approche par reportage. C'est la raison pour laquelle on choisit un texte qui ne crée par d'aiguillages mentaux provoquant un centre d'attention chez le téléspectateur, et que l'on trie les images au montage en éliminant des plans magnifiques, intéressants mais hors de propos, susceptibles d'entraîner le public, dans une émotion, un souvenir, un raisonnement autre.

11 Tout cela aboutit à différents compromis et à une sorte de fiction qui se déroule en trois temps :

- le temps de tournage différent du temps réel. Si un événement se déroule en 3 heures, le journaliste et le cadreur tourneront par exemple 15 minutes pour leur émission ;

- le temps du montage qui réduira encore la durée du reportage ;

- et finalement le temps du commentaire qui vient en complément du film monté.

Les compromis s'effectuent à partir des exigences des différents métiers, celles des caméramen qui raisonnent en termes cinématographiques, celles des monteurs et des journalistes qui ont avant tout le souci de l'information. C'est l'angle du reportage qui doit tout dominer, le tournage, le montage, le texte, la qualité des interviews, le choix des témoins (avec une importance toute particulière donnée aux images qui doivent amener la présentation du sujet).

Pour la raison que si quelqu'un regarde la télévision, c'est l'image qui l'emporte. La pire télévision en matière de reportage est celle qui propose des images supports ou prétextes plaquées artificiellement sur une bande sonore : quand la télévision se met à faire de la radio... La mémoire visuelle étant la plus forte, on retient davantage ce qui est montré, que ce qui est dit. 


\section{DÉBAT}

15 Dans l'exemple de reportage que nous avons visionné ensemble sur l'affaire Grégory ; il y avait un seul angle qui était illustré par la phrase " saura-t-on jamais ce qui s'est passé »; le journaliste a relevé dans cette séquence d'informations toutes les incertitudes, les flous qui jalonnent cette affaire.

16 Question: Le montage au début du reportage, en fondu enchaîné sur l'eau trouble de la Vologne illustre-t-il aussi ce choix journalistique?

17 B. Marquetti : Oui, en même temps il indique une notion de durée, du temps qui s'est écoulé entre la disparition de l'enfant Grégory et l'assassinat de son oncle, c'est aussi une façon de dramatiser et de rappeler que l'on a retrouvé le corps du petit garçon dans cette Vologne.

L'eau dans ce cas de figure constitue le fil directeur du reportage qui était difficile à rendre intéressant car l'affaire était extrêmement connue et banalisée.

18 Question : L'exemple de ce reportage est intéressant mais que se passe-t-il quand le journaliste n'a pas d'images à sa disposition pour parler d'un événement?

19 B. Marquetti : Dans ce cas, ce sont des images d'archives, de rappel ou les images-prétextes dont je parlais plus haut, ou bien encore des images fabriquées par ordinateur qui vont soutenir l'information.

20 Question: Ne pourrait-on pas échapper à la règle que vous nous avez indiquée de l'adéquation toutes images pour permettre justement parfois au public de réfléchir et non d'avoir sans cesse l'angle ou le point de vue du journaliste?

21 B. Marquetti : Ce n'est pas une question de point de vue de la part du journaliste. Dans la masse d'informations qui tombe chaque jour, un certain nombre d'entre elles sont choisies, hiérarchisées, il faut qu'elles soient reçues, comprises par le public.

Pour qu'elles le soient, il faut mettre en oeuvre ce que j'ai dit à propos de l'image et du son et choisir une idée forte pour la faire passer. Ce n'est pas une opinion, mais un choix opéré à partir de critères professionnels et discuté en conférence de rédaction.

22 Question: A propos des compromis dont vous parliez tout à l'heure entre les métiers, l'arbitrage vient-il parfois des gens d'images, caméramen ou monteurs?

B. Marquetti : Le commentaire se construit le plus souvent dans la salle de montage. Et il y a parfois des effets pervers extraordinaires. Le journaliste cherche des mots ou des expressions qui lui plaisent et pousse le monteur parfois à trouver l'image qui leur correspond, c'est une erreur de chercher des images, alors que l'on peut si facilement changer les mots. Je connaissais un chef monteur qui disait " quand le journaliste dit : "ce faisan 'je mets un faisan à l'image mais quand il dit: 'le soir tombe ' je n'ai jamais rien trouvé... ”.

Question : Quelles évolutions du journal TV sont prévisibles dans ce contexte de concurrence entre les chaînes privées et publiques?

B. Marquetti : Il y a dix ans le journal de 20 h était à $20 \%$ ce que les radios avaient donné le matin (sur Europe 1 et presque partout), et l'on avait une journée pour fabriquer l'image, la construire ou l'acheter ailleurs, les gens découvraient le journal de $20 \mathrm{~h}$ avec les images de ce qu'ils savaient déjà sur l'actualité. A cette époque on disait, il faut rattraper les radios! là-dessus ont surgi diverses avancées technologiques, ou prétendues telles: les vidéocassettes, le journal électronique, la Bétacam, le super 8. Et nous avons tout misé sur la rapidité que permettaient ces moyens. Maintenant c'est l'inverse, vous pouvez constater que $90 \%$ de ce qui est dit le matin à la radio, nous 
l'avons montré la veille. A quel prix pour la qualité des images!

On a placé le challenge, là où il ne devait pas être, et, nous vivons souvent une absurdité, l'image standard remplace celle que l'on allait chercher et qui coûtait plus cher. La privatisation a multiplié les contraintes économiques et l'on cède à une facilité au détriment de l'image. Peut-être va t-on vers une évolution du journal télévisé de type visiophone, quelqu'un va parler et on aura son image où qu'il soit dans le monde...

\section{RÉSUMÉS}

Compte-rendu de l'interview du journaliste Benoît Marquetti sur le rapport entre images et textes dans les reportages. L'interview est suivie du compte-rendu d'un débat avec Benoît Marquetti sur le même sujet.

\section{INDEX}

Mots-clés : image, texte, reportage, télévision

Keywords : image, text, report, televison

\section{AUTEUR}

\section{BERNARD MARQUETTI}

Bernard Marquetti, Antenne 2 